\title{
Efficacy of Nitrofurantoin in Catheter Associated Urinary Tract Infection
}

\author{
Nikita Birhman $^{1 *}$, Sneha Mohan ${ }^{2}$ and Dalip K Kakru ${ }^{3}$ \\ ${ }^{1}$ MSc. Medical Microbiology, Department of Microbiology, School of Medical Sciences \\ and Research, Sharda University, Greater Noida, UP, India \\ ${ }^{2}$ Assistant Professor, Department of Microbiology, School of Medical Sciences and \\ Research, Sharda University, Greater Noida, UP, India \\ ${ }^{3}$ Professor, Department of Microbiology, School of Medical Sciences and Research, \\ Sharda University, Greater Noida, UP, India \\ *Corresponding Author: Nikita Birhman, MSc. Medical Microbiology, Department \\ of Microbiology, School of Medical Sciences and Research, Sharda University, Greater \\ Noida, UP, India.
}

Received: April 29, 2020

Published: June 01, 2020

(C) All rights are reserved by Nikita

Birhman., et al.

\begin{abstract}
Introduction: Catheter-associated urinary tract infection (CAUTI) is one of the most common causes of hospital-acquired infections. Nitrofurantoin, a broad-spectrum bactericidal antibiotic, is frequently used to treat UTI, including the catheter associated urinary tract infections.

Materials and Methods: 300 catheterized urine samples were received in the bacteriological laboratory during the study period. The specimens were processed as per the standard bacteriological procedures.

Results: Out of 300 samples included in this study a total of 76 strains were isolated and identified on the basis of culture characteristics, Gram's stain and biochemical reactions. The isolates were subjected to antimicrobial susceptibility and efficacy of nitrofurantoin was analysed in various organism.

Conclusion: In the present study, we found that Nitrofurantoin (NIT) drug was more effective followed by other antibiotics. This is a welcome finding in times of increasing antibiotic resistance and is a reason enough to suggest prophylactic use of Nitrofurantoin in the health care settings when in dwelling catheters become a necessity.

Keywords: Bacteriological Profile; CAUTI; UTI
\end{abstract}

\section{Introduction}

Any part of the urinary system involves in the urinary tract infection (UTI) - kidneys, ureters, bladder and urethra. Most infections involve the bladder and the urethra. Many urinary infections resolve spontaneously, but others can progress to destroy the kidney [1].

The urinary tract is the commonest site of nosocomial infections, accounts to more than $80 \%$ of infections [2]. Catheter associated urinary tract infection is one of the most common cause of hospital acquired infections [3]. It is defined by the Centers for Disease Control and prevention (CDC) as any urinary tract infection in a patient who had an indwelling catheter in place at the time of or within 48 hours prior to onset of infection [4].
Urinary tract infection in 20 - 50\% women happen at some time or the other in their life and a significant number have recurrent infections [1]. Although majority of infections are acute, they contribute to a significant amount of morbidity and health care expenditure in the population [1]. Infection of urinary tract is a common distressing and occasionally life-threatening condition [5]. Almost $40 \%$ of all health care associated infections, urinary tract infections are the foremost cause of infections; out of these, a bulky proportion, $80 \%$, involve catheter associated urinary tract infections [6].

Microbiological profile and antimicrobial sensitivity pattern of CAUTI vary considerably from time to time. Gender, age, malnutrition, low socioeconomic conditions with poor hygiene, structural 
and functional abnormalities of urinary tract infection are many predisposing factors for developing CAUTI [7]. Escherichia coli, Klebsiella, Enterococci, Enterobacter, Acinetobacter, Pseudomonas and Proteus are common pathogens that colonize urinary tract. The relative frequency of the pathogens varies depending upon age, sex, catheterization and hospitalization [8]. Antimicrobial resistance among bacterial pathogens is of worldwide concern [5]. Thus, it is necessary to identify the bacterial spectrum and antimicrobial susceptibility of the uro-pathogens [5]. Broad spectrum bactericidal antibiotic is Nitrofurantoin and is effectively used to treat urinary tract infections. The bacterial laboratory plays an important role in the investigations of the patients suspected of catheter associated UTI [1].

\section{Materials and Methods}

This study was conducted in the Department of Microbiology, School of Medical Science and Research, Sharda Hospital and Sharda University, Greater Noida. This study was conducted for a period of 6 months (1 May - 31 October). 300 catheterized urine samples were received in the bacteriological laboratory during the study period. The specimens were processed as per the standard bacteriological procedures available [6]. In the case of undue delay, the samples were stored at $4^{\circ} \mathrm{C}$.

\section{Direct microscopy}

Gram Staining was done which gave an idea of whether the bacteria were gram-positive or gram negative [7].

Urine culture

The culture media used were Cystine-Lactose-Electrolyte-Deficient Agar (CLED). The media was made and sterilized at $121^{\circ} \mathrm{C}$ for 15 minutes at a pressure of 15 psi in an autoclave. After inoculation, the plates were incubated aerobically at $37^{\circ} \mathrm{C}$ for $24 \mathrm{hrs}$ for bacterial growth.

\section{Automated systems for bacterial identification}

VITEK 2 is an automated system used for identification and antimicrobial susceptibility testing (AST) of bacteria and yeast [7]. Separate cards were available for gram-negative, gram-positive bacteria, fastidious bacteria and yeasts [7]. The results of identification were usually available within $4-6$ hours and AST within 16 - 18 hours [7].

\section{Interpretation}

After 24 hours each plate was examined and growth zones were measured to the nearest millimeter, using sliding caliber held at the back of the inverted media plate. The Petri dish was held a few inches above a black, non-reflecting background and illuminated with reflected light. The inhibition zone margins were taken as the area showing no obvious, visible growth that could be detected with the unaided eye. Faint growth of tiny colonies, detected only with a magnifying lens at the edge of the zone of inhibited growth, was ignored. The results were reported as sensitive or resistant to the agents that had been tested according to the CLSI interpretation criteria (2019).

\section{Results}

Out of 300 samples, 76 (25.33\%) samples showed the growth of culture positivity and 224 showed no growth.

Distribution of pathogenic organisms in samples: A total number of $69(90.78 \%)$ isolates were infected with bacterial infection and $7(9.22 \%)$ isolates were infected with fungal infection. Out of 69; 39 (56.53\%) were E. coli, 6 (8.7\%) were Acinetobacter spp., 2 (2.9\%) were Klebsiella spp., 7 (10.14\%) were Citrobacter spp., 3 (4.34\%) were Proteus spp., 1 (1.45\%) were Staphylococcus aureus, 4 (5.79\%) were Enterococcus spp., 7 (10.14\%) were Pseudomonas spp.

Antibiotic susceptibility pattern of CAUTI causing uropathogen

\section{Escherichia coli}

As an individual agent, Colistin was found to be most effective (100\%), followed by nitrofurantoin (89\%), meropenem (84\%) and imipenem (79.48\%). Ampicillin and Cefuroxime (74\%) were the most resistant drugs followed by Cefepime (71\%) and Norfloxacin (69\%).

\section{Acinetobacter spp.}

Amikacin (100\%), Azithromycin (100\%), Amoxicillin/clavulanic (100\%), Cefotaxime (100\%), Chloramphenicol (100\%), Ceftriaxone (100\%), Ciprofloxacin (100\%), Colistin (100\%) and Norfloxacin $(100 \%)$ and Nitrofurantoin (50\%) were the drugs found to be effective. 


\section{Proteus spp.}

As there was only 1 isolate of Proteus spp., the most effective drugs were found to be Amikacin, Colistin, Aztreonam, Azithromycin, Ampicillin/sulbactam, Chloramphenicol, Ciprofloxacin, Nitrofurantoin and Tetracycline.

\section{Citrobacter spp.}

Amongst all the antibiotics, Azithromycin (100\%), Colistin (100\%) and Ceftazidime (100\%) were found to be most effective against Citrobacter. They were followed by Imipenem (66.66\%), Aztreonam (66.66\%), Amoxicillin/clavulanic (66.66\%), Chloramphenicol (66.66\%), Ciprofloxacin (66.66\%), Cefepime (66.66\%), Meropenem (66.66\%) and Piperacillin/tazobactam (66.66\%) and Nitrofurantoin(33.33\%).

\section{Klebsiella spp.}

Amongst all the antibiotics, Aztreonam, Amoxicillin/clavulanic, Colistin, Ampicillin/sulbactam, Ticarcillin/clavulanic (100\%) were found to be most effective against Klebsiella. They were followed by Amikacin (85\%), Azithromycin (85\%), Imipenem (85\%), Nitrofurantoin (85\%) and Piperacillin/tazobactam (85\%).

\section{Pseudomonas spp.}

Amongst all the antibiotics, Amikacin, Aztreonam, Gentamicin, Colistin, Piperacillin, Meropenem, Nitrofurantoin, Ticarcillin/ clavulanic were found to be the best drug showing sensitivity of $100 \%$.

\section{Staphylococcus aureus}

There were 6 classes of antibiotics tested for antibiotic susceptibility against Staphylococcus aureus. Gentamicin and Vancomycin found the most effective drug followed by Ciprofloxacin, Nitrofurantoin, Tetracycline and Clindamycin.

\section{Enterococcus spp.}

Antibiotic sensitivity pattern of enterococcus was tested against penicillin, vancomycin, linezolid, levofloxacin, erythromycin, cefoxitin, nitrofurantoin, norfloxacin and gentamicin. It was observed that nitrofurantoin and vancomycin was $100 \%$ effective drug.

\begin{tabular}{|l|c|}
\hline Organisms causing CAUTI & $\begin{array}{c}\text { Percentage of sensitivity of } \\
\text { nitrofurantoin }\end{array}$ \\
\hline Escherichia coli & 89.74 \\
\hline Enterococcus spp. & 100 \\
\hline Staphylococcus aureus & 75 \\
\hline Pseudomonas spp. & 100 \\
\hline Klebsiella spp. & 85.79 \\
\hline Citrobacter spp. & 33.33 \\
\hline Proteus spp. & 50 \\
\hline Acinetobacter spp. & 50 \\
\hline
\end{tabular}

Table 1: Percentage showing the sensitivity of NIT among organisms causing CAUTI.

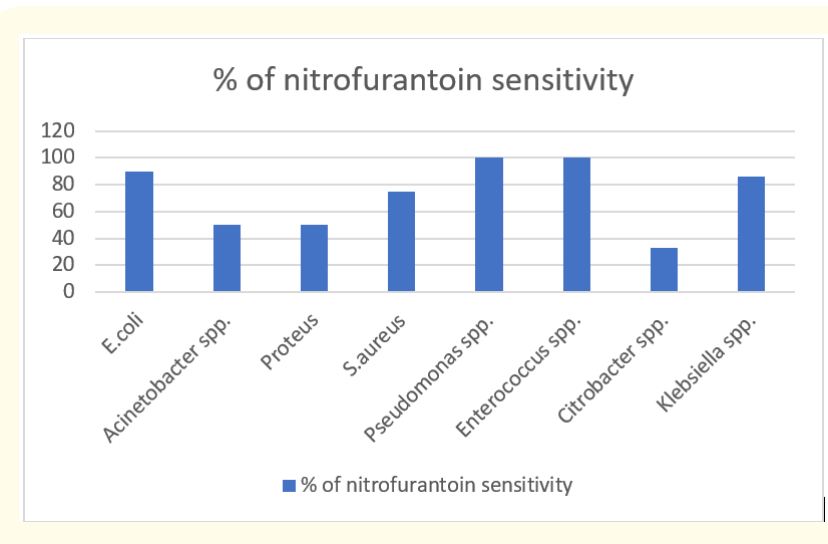

Figure

\section{Discussion}

Out of the 300 patients analysed, 76 (25.33\%) of them developed CAUTI, which is similar to studies done by Kulkarni., et al. [9], Bhatia., et al. [10], Bagchi., et al [11]. From 76 (25.33\%) isolates, bacterial pathogens were 69 (90.78\%) and 7 (9.22\%) were fungal. Escherichia coli was the most common organism isolated followed by other organisms such as Proteus, Klebsiella spp., Pseudomonas spp., Acinetobacter spp., Citrobacter spp., Enterococcus and Staphylococcus aureus. The sensitivity of all the organisms was assessed based on CLSI guidelines. Most of the gram positives and gram negative bacilli showed complete sensitivity to nitrofurantoin drugs. This result was also comparable to the study done by Naveen G., et al [2]. Nitrofurantoin is active against most common uropathogens 
including E.coli, Citrobacter spp., Staphylococcus spp., Enterococcus spp. This was also comparable to the study done by Shakti L., et al. While the rate of nitrofurantoin in resistance was lower than any of the other drugs, some studies have reported high levels of resistance. Nitrofurantoin has been used successfully for treatment and prophylaxis of catheter associated urinary tract infection. With the emergence of antibiotic resistance, nitrofurantoin has become the choice of agent for treating UTIs caused by multi-drug resistant pathogens.

\section{Conclusion}

In the present study we found that Escherichia coli was the major reason of infection but the organism incidentally was more susceptible to Nitrofurantoin followed by other antibiotics. Nitrofurantoin remains the only available alternative with almost equivalent activity against with CAUTI causing organisms and had high susceptibility rate among other antibiotics for CAUTI. This is a welcome finding in times of increasing antibiotic resistance and is a reason enough to suggest prophylactic use of Nitrofurantoin in the health care settings when in dwelling catheters become a necessity. To provide optimum use and to avoid misuse and overuse of this drug, culture, and susceptibility testing is the need of the time to preserve it for next generation. Also, as several studies suggest, the antimicrobial Nitrofurantoin might be a suitable antimicrobial agent to coat catheters so that whatever probable chances of CAUTI are there, will also get minimised.

\section{Bibliography}

1. SK Hasan A., et al. "Laboratory diagnosis of urinary tract infections using diagnostics tests in adult patients". International Journal of Research in Medical Sciences 2.2 (2014): 415.

2. Naveen G., et al. "Bacteriological study of Catheter Associated Urinary Tract Infection in a Tertiary Care Hospital". International Journal of Current Microbiology and Applied Sciences 5.9 (2016): 640-644.

3. Ramesh A., et al. "Microbiological profile, comorbidity, incidence and rate analysis of catheter tract infections in adult intensive care unit". Indian Journal of Microbiology Research 5.1 (2018): 38-43.

4. Bagchi I., et al. "Microbiological evaluation of catheter associated urinary tract infection in a tertiary care hospital". People's Journal of Scientific Research 8.2 (2015): 23-29.
5. Patil HV and Patil VC. "Clinical, Bacteriology Profile and Antibiotic Sensitivity Pattern of Catheter Associated Urinary Tract Infection at Tertiary Care Hospital". International Journal of Health Sciences and Research 8.1 (2018): 25-35.

6. Barad PS and DL Barate. "Prevalence and Antibiotic susceptibility pattern of Bacteria isolated from catheter Associated Urinary Tract Infection". Bioscience Discovery 9.1 (2018): 1-7.

7. Jayasukhbhai D., et al. "Study of incidence and risk factors of urinary tract infection in catheterised patients admitted at tertiary care hospital". International Journal of Research in Medical Sciences 3.12 (2015): 3808.

8. Maharajan G., et al. "Catheter Associated Urinary Tract Infection and Obstinate Biofilm Producers". Canadian Journal of Infectious Diseases and Medical Microbiology (2018): 7624857.

9. Kulkarni SG., et al. "Profile of urinary tract infection in indwelling catheterized patients". IOSR Journal of Dental and Medical Sciences 13.4 (2014): 132-138.

10. Bhatia N., et al. "Urinary catheterization in medical wards". Journal of Global Infectious Diseases 2.2 (2010): 83-90.

11. Bagchi I., et al. "Microbiological Evaluation of Catheter Associated Urinary Tract Infection in a Tertiary Care Hospital". People's Journal of Scientific Research 8.2 (2015): 23-29.

\section{Assets from publication with us}

- Prompt Acknowledgement after receiving the article

- Thorough Double blinded peer review

- Rapid Publication

- Issue of Publication Certificate

- High visibility of your Published work

Website: www.actascientific.com/

Submit Article: www.actascientific.com/submission.php

Email us: editor@actascientific.com

Contact us: +919182824667 